Infusionstherapie 1973-74;1:583

\title{
Impressum, Vol. 1, No. 8, 1973-74
}

S. Karger Verlag für Medizin und Naturwissenschaften GmbH, 8034 Germering, Postfach 2 Chirurgie

Anästhesie

Nephrologie

Innere Medizin

Pharmazie

Pädiatrie

Innere Medizin

Pharmakologie

Innere Medizin

Exp. Chirurgie

Anästhesie

Innere Medizin

Anästhesie

Neurochirurgie

Chirurgie

Biochemie

Pädiatrie

Innere Medizin

Anästhesie

Chirurgie

Anästhesie

Urologie

Anästhesie

Ernährungsphysiologie

Urologie

Chirurgie

Chirurgie

Herausgeber

Prof. Dr. H. Reissigl, W. Hofrat

Vorstand des Zentralinstituts für Bluttransfusion und Immuno-

logische Abteilung der Universitätskliniken Innsbruck, an der

Chirurgischen Universitätsklinik, Anichstraße 35, A-6020

Innsbruck

Prof. Dr. H. Grobecker

Zentrum für Pharmakologie, Abt. Ill, Johann-Wolfgang-

Goethe-Universität, Theodor-Stern-Kai 7, D-6000 Frankfurt/

Main 70

Prof. Dr. U. Henneberg 
Geschäftsführender Direktor des Instituts für Anästhesiologie im Klinikum Steglitz der Freien Universität Berlin, Hindenburg-damm 30, D-1 Berlin 45

Wissenschaftlicher Beirat

Verwaltung

Chirurgie

Anästhesie

Anästhesie

Chirurgie

Transfusionsmedizin

Anästhesie

Chirurgie

Bluttransfusion

Innere Medizin

Bluttransfusion

Biochemie

Pharmazie

Nephrologie

Gynäkologie

Anästhesie

Hämatologie

Pharmakologie

Innere Medizin

Innere Medizin

E.H.Abel, Stade Dr. W. Bayerl, Bonn Prof. Dr. H. Bergmann, Linz Dr. H. Boll, Hamm Prof. Dr. C. Burri, Ulm Prof. Dr. H. Busch, Hamburg Prof. Dr. W.Dick, Ulm Prof. Dr. R. Dohrmann,

Berlin Prof.Dr. H.Egli, Bonn Prof. Dr. M. Eggstein, Tubingen Prof. Dr. C. C. Ehrich, New York Prof. Dr. H.Förster, Frankfurt Dr. P. Frank, Würzburg Prof. Dr. H. E. Franz, Ulm Prof. Dr. V.

Friedberg, Mainz Prof. Dr. M. Gemperle, Genf Prof. Dr. A. Hässig, Bern Prof. Dr. O.

Heidenreich, Aachen Prof. Dr. A. Heidland, Würzburg Priv. Doz. Dr. P. U. Heuckenkamp,

München

Prof. Dr. B. Hölscher, Berlin

Prof. Dr. G. Hossu, Zurich

Dr. K.F. Kopp, München

Prof. Dr. F. Krück, Homburg/Saar

D $\gamma$. H.J.Kümmell, Melsungen

Priv. Doz. Dr. V. O. Lang, München

Prof. Dr. H. G. Lasch, Gießen

Prof. Dr. A. Lindner, Wien

Prof. Dr. A. Linke, Ludwigshafen

Prof.Dr. P. Lundsgaard-Hansen, Bern

Prof. Dr. H. Lutz, Mannheim

Priv. Doz. Dr. J. Papenberg, Heidelberg

Priv. Doz. Dr. K. Peter, Mannheim

Prof. Dr. H. W. Pia, Gießen

Prof. Dr. G Rodewald, Hamburg

Prof. Dr. L. Róka, Gießen 
Prof. Dr. E. Rossi, Bern

Prof. Dr. G. Schettler, Heidelberg

Dr. H. Schmidt, Frankfurt

Prof. Dr. I. Staib, Darmstadt

Prof. Dr. H. Stoeckel, Bonn

Prof. Dr. W. Vahlensieck, Bonn

Prof. Dr. K. Wiemers, Freiburg

Prof. Dr. A. Wretlind, Stockholm

Prof. Dr. M.Ziegler, Heidelberg

Prof. Dr. W. E. Zimmermann, Freiburg

Prof. Dr. R.X.Zittel, Ludwigshafen

Schriftleitung

Hans-Dieter Viering

Waldschmidstraße 8, D-8130 Starnberg am See

Tel.: 08151-6866

Lektorat: Rotraut Schwoerer, Starnberg am See

Herstellung: Werner Zuckschwerdt, Starnberg am See

Satz und Druck: Buch- und Offsetdruckerei Walter Biering KG, 8 München 40

Erscheinungsweise

»Die Infusionstherapie« erscheint zweimonatlich; pro Jahr er-scheint 1 Band zu je 6 Heften; jedes Heft hat ungefähr 80 Seiten Inhalt. Jahrgang 1 deckt sich nicht mit dem Kalenderjahr. Er hat einen von der Regel abweichenden Umfang von 8 Heften, die in Abständen von 2 Monaten im Zeitraum Oktober 1973 bis Dezember 1974 erscheinen.

Abonnementspreis

Bezugspreis für Jahrgang 1, 1973/74 (8 Hefte) DM 92,-/US \$ 35,-/SFr 96,-/ÖS 690 -

(Abonnenten, die bis Februar 1974 bis-lang nur 6 Hefte = DM 69,-- belastet erhielten, erhalten für die Erweiterung des Jahrganges um 2 Hefte eine Nachbelastung von DM 23,-). 1 Einzelheft kostet DM 14,-/US \$ 4,50/SFr 14,40/ OS 105,-, einschließlich MwSt., zuzüglich Postgebühren. Der Abonnementspreis ist im voraus zahlbar. Die Lieferung der Zeitschrift läuft weiter, wenn sie nicht spätestens 4 Wochen vor Abschluß eines Bandes abbestellt wird.

Sonderhefte sind nur in beschränkter Auflage vorrätig. Der Preis eines Sonderhefts ist abhängig vom Umfang. Abonnenten erhalten die Sonderhefte zu einem um 20\% vom Ladenpreis ermäßigten Vorzugspreis.

Abonnements

Abonnementsbestellungen können bei jeder Buchhandlung, bei der Post oder direkt beim Verlag aufgegeben werden: Bundesrepublík Deutschland/ Österreich: S. Karger GmbH, Postfach 2, D8034 Germering/München, Tel.: 089-844021, Telex: 524865 D, Postscheckkonto: München 40080-807 Schweiz: S. Karger AG, Arnold-Böcklin-Straße 25, CH-4011 Basel, Tel: 061390880, Telex $62652 \mathrm{CH}$

Frankreích: S. Karger S.A., 42bis, Boulevard de la Tour-Mau-bourg, F-75007 Paris

England: John Wiley \& Sons Ltd., Baffins Lane, Chichester USA: Albert J.Phiebig, Inc., P.O.

Box 352, White Plains, New York, NY 10602

Anzeigen

MPV med. pharm. Verwaltungsgesellschaft mbH \& Co. KG,

Schießstättstraße 14, Postfach 1547, D-8130 Starnberg, Telefon 
(08151)13299.

Gültig ist die Preisliste Nr. 2 vom 1. 3.1974.

Der Gesamtauflage der Zeitschrift sind beigefügt:

eine Bestellkarte des Verlages S. Karger für die Zeitschrift und ein Einhefter der Firma

Pfrimmer, Erlangen. 